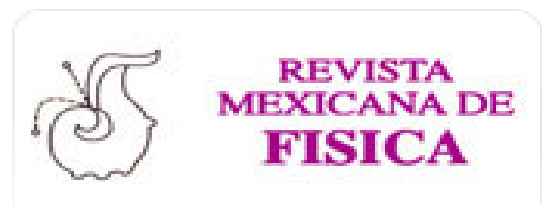

Revista Mexicana de Física

ISSN: 0035-001X

rmf@ciencias.unam.mx

Sociedad Mexicana de Física A.C.

México

Reyes-Ramírez, I.; Fernández-Rosales, I.; Guzmán-Vargas, L.

Scaling differences of heartbeat excursions between wake and sleep periods

Revista Mexicana de Física, vol. 58, núm. 1, junio-, 2012, pp. 91-95

Sociedad Mexicana de Física A.C.

Distrito Federal, México

Available in: http://www.redalyc.org/articulo.oa?id=57030391016

How to cite

Complete issue

More information about this article

Journal's homepage in redalyc.org

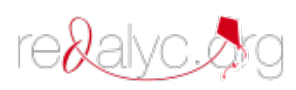

Scientific Information System Network of Scientific Journals from Latin America, the Caribbean, Spain and Portugal Non-profit academic project, developed under the open access initiative 


\title{
Scaling differences of heartbeat excursions between wake and sleep periods
}

\author{
I. Reyes-Ramírez ${ }^{a, b}$, I. Fernández-Rosales ${ }^{a}$, and L. Guzmán-Vargas ${ }^{a}$ \\ ${ }^{a}$ Unidad Profesional Interdisciplinaria en Ingeniería y Tecnologías Avanzadas, \\ Instituto Politécnico Nacional, Av. IPN No. 2580, L. Ticomán, México D.F. 07340, México. \\ ${ }^{b}$ Escuela Superior de Física y Matemáticas, Instituto Politécnico Nacional, \\ Ed. 9 UP Zacatenco, México D.F. 07340, México.
}

Recibido el 23 de Marzo de 2010; aceptado el 27 de Abril de 2011

In this work, we study sequences of excursions from heart interbeat time series. An excursion is defined as the time employed by a walker to return to its mean value. Scaling properties of excursions during wake and sleep periods from two groups are compared: 16 healthy subjects and 11 patients with congestive heart failure (CHF). We find that the cumulative distributions of excursions for both groups follow stretched exponential functions given by $g(x) \sim e^{-a \tau^{b}}$ with different fitting parameters $a$ and $b$. Next, we explore changes in the distributions of excursions when considering (i) a shifted mean value to define an excursion and (ii) the sum of the $k$-th excursion successor. Finally, the presence of correlations in the excursions sequences is evaluated by means of the detrended fluctuation analysis.

Keywords: Excursions; distributions; correlations.

En este trabajo se estudian series de tiempo de secuencias de excursiones de latido cardiaco. Una excursión se define como el tiempo que le toma a un caminante que se desvia de su valor medio, retornar a él. Se comparan las propiedades de escalamiento de las excursiones interlatido, durante los periodos de sueño y vigilia de dos grupos, uno de 16 sujetos sanos y el otro de 11 pacientes con falla cardiaca congestiva. Se encuentra que las distribuciones cumulativas de ambos grupos se describen bien mediante exponenciales extendidas con diferentes parámetros de ajuste. Enseguida se exploran los cambios en las distribuciones de las excursiones, generados por, (i) un desplazamiento en el valor medio al definir las excursiones, y (ii) la suma de la k-esima excursión subsecuente. Finalmente, se evalúan las correlaciones en las secuencias de excursiones mediante un análisis de fluctuaciones sin tendencia.

Descriptores: Excursiones; distribuciones; correlaciones.

PACS: 87.19.Hh; 87.10.+e; 89.20.-a

\section{Introduction}

In past decades, many studies have focused on statistical properties of heartbeat interval time series. These findings have permitted to characterize healthy heartbeat dynamics as fluctuations with $1 / f$ behavior with long-range correlations and a broad multifractal spectrum [1-3]. An important aspect of the interbeat variability is that healthy systems have complex self-regulating mechanisms operating over multiple time scales and may generate signals that have scaling properties. Recent research focused on evaluation of complexity in heart rate variability have revealed that some fractal and scaling features which characterize healthy dynamics suffer changes under pathologic conditions [1,4-8]. Another important characteristic of heartbeat time series is the nonstationarity related with a large number of control mechanisms of the heart and external stimuli. However, when the interbeat sequences are observed locally, one can roughly define a local mean value. From a physiologic point of view, the presence of local stationary segments can be understood as the capability of the system to preserve an approximated constant value but for a limited period of time. It is also argued that, according to the homeostasis principle, biological systems tend to maintain a constant output in spite of continual perturbations [9]. In fact, a previous study to detect local stationary segments of heartbeat interval time series revealed that the distribution of these stationary segments follows a power law behavior and the scaling exponent, which characterizes the distribution, is the same for healthy and heart failure groups [10]. Here we focused our attention in the scaling properties of excursions, which are defined as the period of time employed by a walker to return to its average value, to evaluate the capability of the system to preserve a mean output value and to compare differences between wake and sleep periods. The problem of the first return time has been studied in contexts like financial index, intermittency, seismic activity and simulated noise [11-14]. There are also important results of zero crossing probabilities for Gaussian long-term correlated data [15]. We recently reported important scaling characteristics of excursions for healthy and pathologic cardiac dynamics [16]. In the present work we address the question if there are changes in the scaling properties of excursions between diurnal and nocturnal periods under healthy and heart failure conditions. The paper is organized as follows: In Sec. 2, we briefly describe the segmentation algorithm and the detrended fluctuation analysis method. In Sec. 3, the results of the statistics of excursions are described. Finally, some concluding remarks are given in Sec. 4.

\section{Methods}

\subsection{The segmentation method}

To detect stationary segments in heartbeat time series, we use the segmentation method proposed by Bernaola-Galván [10]. 


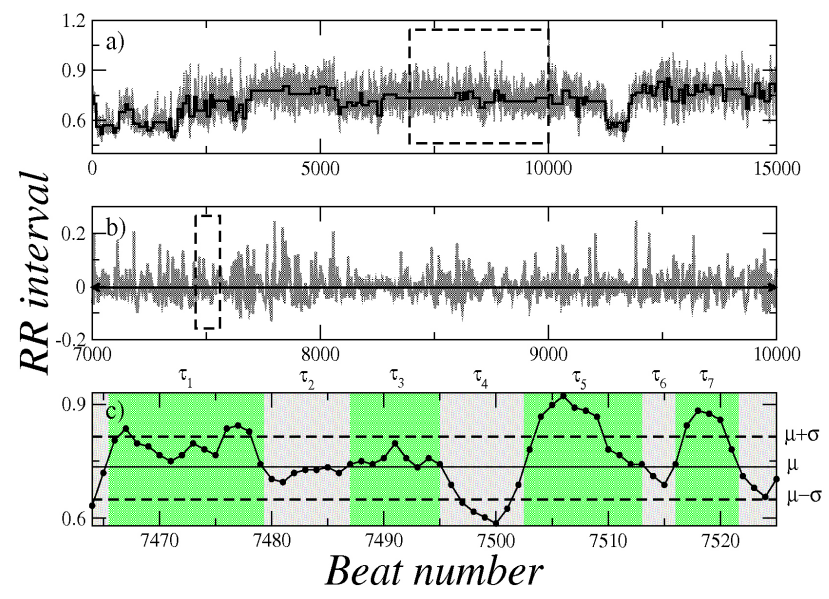

FIGURE 1. Representative case of the segmentation procedure for a (a) nonstationary signal from a healthy subject. (b) As in (a) but for a magnification of the interval in (a). (c) Magnification of (b) to illustrate the excursion identification.

The method consists in considering a slider pointer to calculate the statistics

$$
t=\frac{\mu_{r}-\mu_{l}}{S_{D}}
$$

where $\mu_{r}$ and $\mu_{l}$ are the mean of the values on the right and left, respectively. $S_{D}$ is the pooled variance given by

$$
S_{D}=\left(\frac{\left(N_{l}-1\right) s_{l}^{2}+\left(N_{r}-1\right) s_{r}^{2}}{N_{l}+N_{r}-2}\right)^{1 / 2}\left(\frac{1}{N_{l}}+\frac{1}{N_{r}}\right)^{1 / 2}
$$

where $s_{l}$ and $s_{r}$ are the standard deviation of the two sets, and $N_{l}$ and $N_{r}$ are the number of points in the two sets. The quantity $t$ is used to separate two segments with a statistically different mean. A significance level is applied to cut the series into two new segments (typically set to 0.95), as long as the means of the two new segments are significantly different from the mean of the adjacent segments $[10,17]$. The process is applied recursively until the significance value is smaller than the threshold or the length of the new segment is smaller than a minimum $\ell_{0}$.

\subsection{Correlations}

A method which is very appropriated to the assessment of correlations in stationary and nonstationary time series is the detrended fluctuation analysis (DFA). This method was introduced to quantify long-range correlations in the heartbeat interval time series and DNA sequences $[18,19]$. The DFA is briefly described as follows: First, we integrate the original time series to get,

$$
y(k)=\sum_{i=1}^{k}\left[x(i)-x_{\mathrm{ave}}\right]
$$

the resulting series is divided into boxes of size $n$. For each box, a straight line is fitted to the points, $y_{n}(k)$. Next, the line points are subtracted from the integrated series, $y(k)$, in each box. The root mean square fluctuation of the integrated and detrended series is calculated by means of

$$
F(n)=\sqrt{\frac{1}{N} \sum_{k=1}^{N}\left[y(k)-y_{n}(k)\right]^{2}},
$$

this process is taken over several scales (box sizes) to obtain a power law behavior $F(n) \sim n^{\alpha}$, with $\alpha$ an exponent, which reflects self-similar and correlation properties of the signal. It is known that $\alpha=0.5$ is associated to white noise (non correlated signal), $\alpha=1$ corresponds to $1 / f$ noise and $\alpha=1.5$ represents a Brownian motion.

\section{Results}

We analyzed two different groups of individuals: 16 healthy subjects and 11 patients with congestive heart failure [20]. For each individual, we considered interbeat sequences with approximately $3 \times 10^{4}$ beats corresponding to 6 hours of ECG records. We selected 6-hours during the day and 6-hours from sleep periods.

\subsection{Distributions of excursions}

A representative case of the segmentation procedure is shown in Fig. 1a. Bernaola-Galván and coworkers [10], reported that the cumulative distribution of stationary segments with local mean follows a power law, $G(>\ell) \sim \ell^{-\delta}$, with $\delta \approx 2.2$ for healthy and heart failure groups [10]. We calculate the excursions return times for each stationary segment with respect to the local mean [16]. More specifically, we identify an excursion with size $\tau$ if $x_{j}>\bar{x}$ and $x_{j+\tau}>\bar{x}$ while $x_{i}>\bar{x}$ for $j<i<j+\tau$ or conversely $x_{j}<\bar{x}$ and $x_{j+\tau}<\bar{x}$ while $x_{i}<\bar{x}$ for $j<i<j+\tau$ (see Fig. 1c). In a recent work, we have reported that the distributions of excursion sequences from stationary segments follow approximately the same functional behavior [16]. This permits to pool the data from all segments to improve the statistics. Our results show that after normalizing the excursions, both groups healthy and CHF are consistent with distributions that follow a stretched exponential behavior given by,

$$
g(\tau) \sim e^{-a \tau^{b}}
$$

where $a$ and $b$ are constants. Specifically, for healthy subjects during diurnal hours, we found $a=1.09 \pm 0.15$ (mean value $\pm \mathrm{SD}$ ) and $b=0.91 \pm 0.11$ (Fig 2a. For night periods, we observed $a=1.41 \pm 0.19$ and $b=0.71 \pm 0.11$. In Fig. 2b, the results for the $\mathrm{CHF}$ patients are presented. The best fit reveals that $a=1.31 \pm 0.23$ and $b=0.77 \pm 0.13$ for day periods whereas for night periods we observed $a=1.44 \pm 0.44$ and $b=0.74 \pm 0.19$. In order to compare the values observed in the distributions, we calculate the characteristic scale associated to the stretched exponential distribution given by

$$
\langle\tau\rangle=\frac{a^{-1 / b}}{b} \Gamma(1 / b)
$$




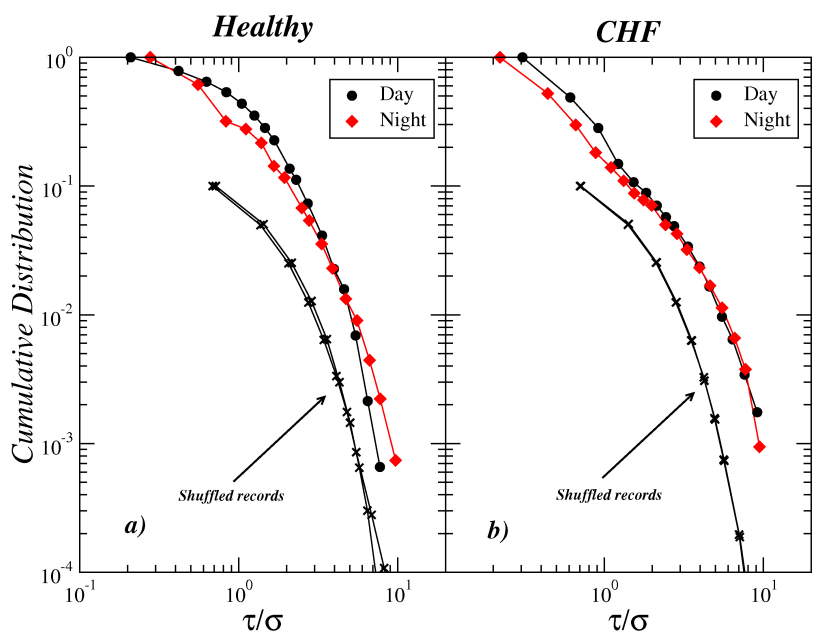

FIGURE 2. Representative cumulative distributions of excursions for wake and sleep periods of (a) a healthy subject and (b) a heart failure patient. We also show the cases of shuffled records, that is, for each segment we shuffled the interbeat time points and then the distribution of excursions was constructed by pooling the data from all segments. For clarity, the distributions were scaled by a factor $1 / 10$.

with $\Gamma$ the Gamma function. Note that for $b=1$, the mean value of an exponential function is recovered. For wake records from healthy data we get $\langle\tau\rangle=0.95 \pm 0.11$, while for $\mathrm{CHF}\langle\tau\rangle=0.84 \pm 0.12$, revealing a fast decay under pathologic conditions. For sleep records from healthy group, the mean value is $\langle\tau\rangle=0.77 \pm 0.10$ whereas for CHF data $\langle\tau\rangle=0.78 \pm 0.24$ (note that both values are quite similar).

In order to evaluate the accuracy of the findings described above, we consider two procedures which affect the distributions: (i) change of the local mean level to define an excursion (see dashed lines in Fig. 1c), (ii) the sum of the $k$-th excursion successor. Concerning (i), we tested the effect of the local mean value over the distribution's parameters. To this end, we repeated the calculations by considering an excursion with respect to a shifted mean value $\mu \pm q \sigma$, with $\sigma$ the standard deviation and $q= \pm 0.1, \pm 0.2, \pm 0.3, \pm 0.4, \pm 0.5$. In Fig. 3 the results of $a$ and $b$ for different values of $q$ are presented. For healthy data from wake periods, we observe that $b$ decreases and $a$ increases as the local mean value is moved upward or downward (see Fig. 3a and its inset) whereas for sleep periods both parameters almost do not change. For heart failure data from wake periods, we also observe that $b$ show a small decreasing and $a$ increases (Fig. $3 \mathrm{~b}$ and its inset) as the local mean value is moved upward or downward whereas for sleep periods both values are almost constant for the shifted mean within the interval $\mu \pm 0.2 \sigma$. We notice that the values of $a$ and $b$ for the CHF group during night periods are close their corresponding values from wake records, revealing that under pathologic conditions wake-sleep changes are less abrupter than for healthy situations.

Regarding the second point, the number of beats from a mean crossing-level (i.e. the starting point of an excursion) and its $k$-th successor is considered; for $k=1$ we get the
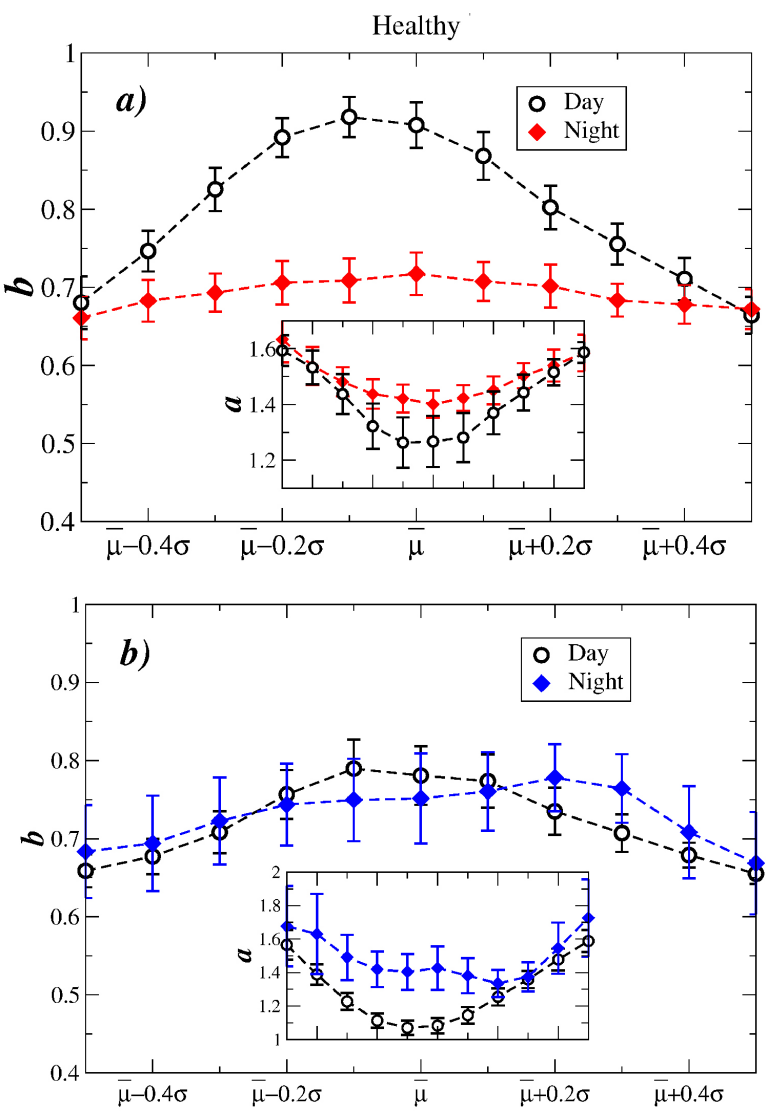

FIGURE 3. Statistics of $a$ and $b$ for distributions obtained from a shifted mean value. Symbols indicate the average value of each parameter and error bars the standard error of the mean. (a) Average values from healthy data during wake and sleep periods. We observe that $b$ decreases as the local mean value is move upward or downward. The opposite behavior is identified for $a$, that is, $a$ increases as the mean value is changed (see the inset). We also remark that $b$ shows a non-symmetrical behavior with respect to the shifted mean value. (b) as in (a) but for CHF records.

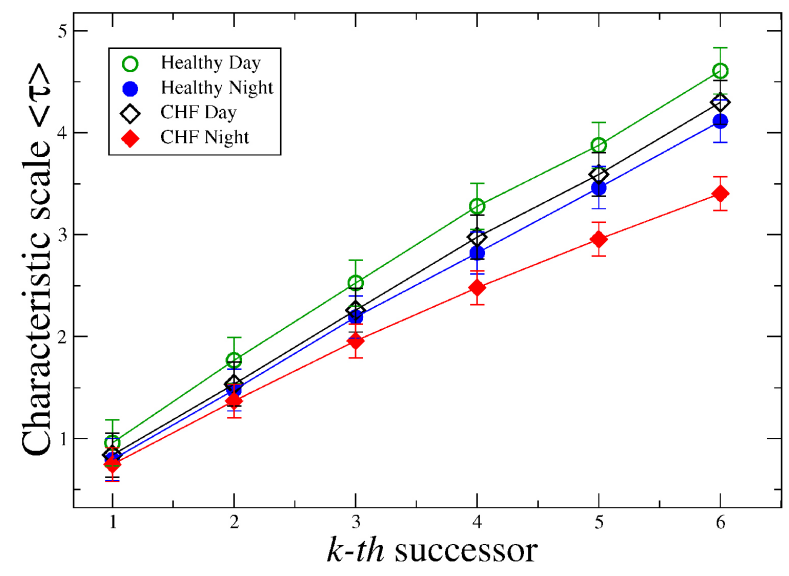

FIgURE 4. Characteristic mean value vs. $k$-th excursion successor for wake and sleep periods. Symbols represent the mean value and error bars the standard error. For healthy and CHF groups, the diurnal mean values are bigger than the sleep mean values for several values of $k$. We note that the healthy mean values from sleep periods are close to the CHF values from wake records. 


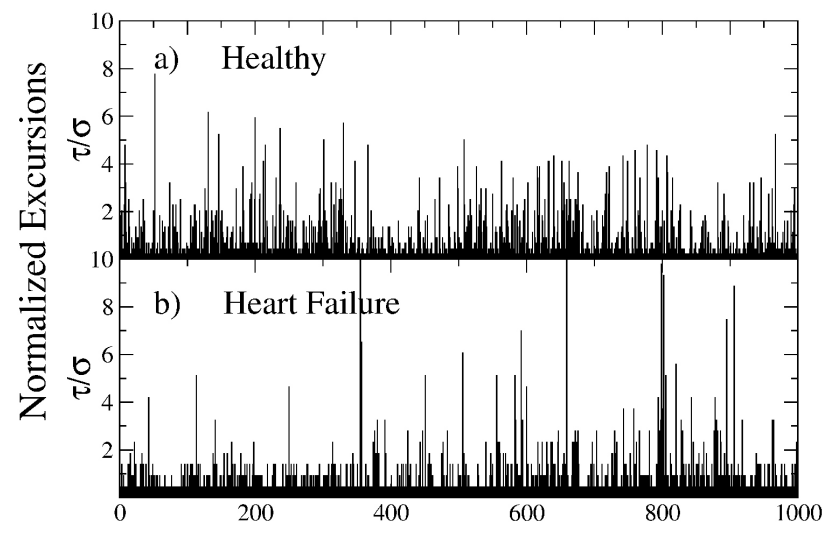

FIGURE 5. Representative sequences of excursions from (a) one healthy subject and (b) one CHF patient during wake periods.

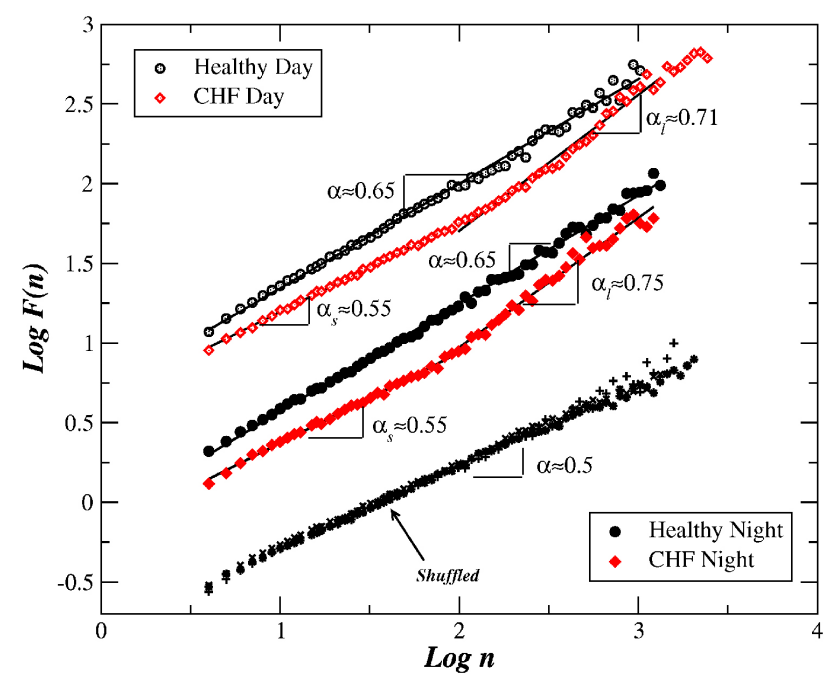

FIGURE 6. Plots of DFA analysis for wake and sleep excursion sequences from a healthy subject and a patient with CHF. For the healthy subject during wake period (open circles) and sleep period (filled circles), a single scaling exponent $\alpha \approx 0.65$ is identified for time scales $10<n<100$. In contrast, a crossover pattern is observed in the CHF patient for day (open diamonds) and night (filled diamonds) records. For short scales the scaling exponent is close to the white noise value $\left(\alpha_{s} \approx 0.55\right)$ whereas for large scales, the excursion sequences display positive correlations $\left(\alpha_{l} \approx 0.7\right)$. As a control, we also show the cases of shuffled records which show a scaling exponent close to the uncorrelated value $\alpha \approx 0.5$.

original excursion definition as those we have studied above. We found that for $k=2,3,4,5$, the cumulative distributions of excursions with $k$-th successor are consistent with a stretched exponential function. We estimate $a$ and $b$ for several values of $k$ to construct the characteristic mean value of the distributions. The results are presented in Fig. 4. We observed that for healthy data from wake periods, the mean value is bigger than the values from sleep records. It is also important to note that healthy mean values from night periods are quite similar to the values from sleep CHF records.

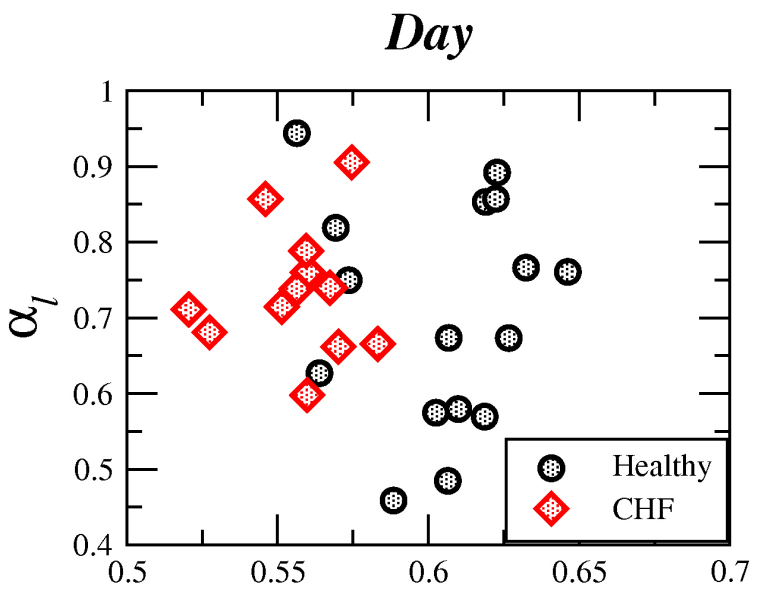

Night

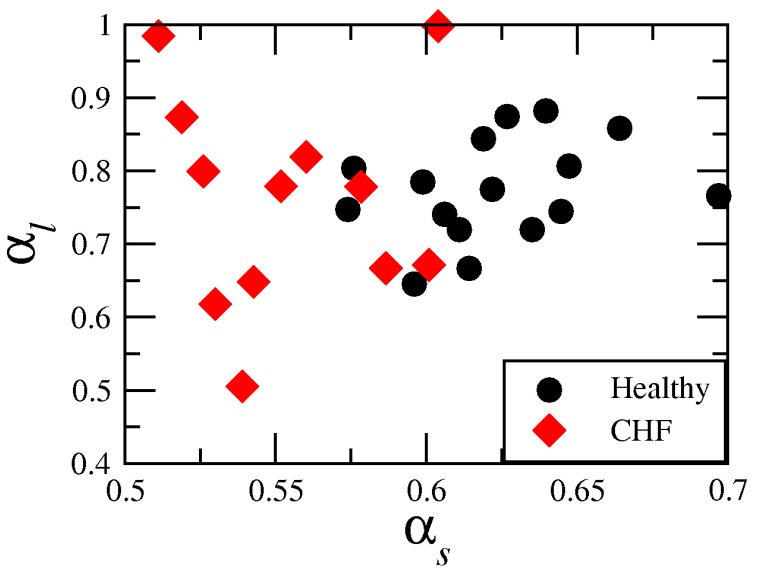

FIGURE 7. Scatter plot of $\alpha_{s}$ vs. $\alpha_{l}$ for wake and sleep excursion sequences from healthy subjects and CHF patients. We estimate $\alpha_{s}$ over short scales $4 \leq n \leq 120$ and $\alpha_{l}$ over large ones $120 \leq n \leq 1000$. A clear separation between the two groups is observed for both periods.

\subsection{Correlations of excursions}

\subsubsection{Detrended fluctuation analysis}

In this Section, we study the memory in the time organization of excursions. Figure 5 shows representative time evolution of excursions from one healthy subject and one CHF patient during wake periods (Fig. 5a and Fig. 5b). We observe that both sequences look different; particularly because of the presence of clusters in healthy data as an indication of memory. We use the DFA method to detect changes in correlations of excursion sequences between sleep and wake periods. As shown in Fig 6, for healthy data from wake periods the scaling behavior along at least for two decades is characterized by the average exponent $\alpha=0.64 \pm 0.04$, while for wake CHF data the scaling is characterized by two regimes; over short scales $\left(4 \leq n \leq 10^{2}\right)$ the average exponent is $\alpha_{s}=0.55 \pm 0.01$ whereas for large scales $\left(10^{2} \leq n \leq 10^{3}\right.$.) the value is $\alpha_{l}=0.71 \pm 0.07$. For sleep periods, we observe that the scaling relation for healthy dynamics is char- 
acterized by the exponent $\alpha=0.64 \pm 0.02$ whereas CHF data are described by the regimes $\alpha_{s}=0.55 \pm 0.03$ and $\alpha_{l}=0.75 \pm 0.13$. We see that in all cases the exponent is bigger than 0.5 , indicating the presence of positive correlations. Important differences are observed when one compares $\alpha_{s}$ and $\alpha_{l}$ for CHF data ( $p$-value $<10^{-3}$ by the Student's test). We remark that for short scales, the average DFA exponent for the healthy group is slightly bigger than the corresponding scaling DFA exponent of CHF group, confirming that the excursion sequences under healthy conditions are more correlated than for pathologic data. It is also important to note that the scaling exponent which characterizes diurnal excursions is almost equal to the exponent from night periods. In order to get a better evaluation of the presence of the crossover in the scaling exponent for both periods, we extracted both scaling exponents $\left(\alpha_{s}\right.$ and $\left.\alpha_{l}\right)$ for healthy data. Fig. 7 shows the scatter plot of the scaling exponents $\alpha_{s}$ vs $\alpha_{l}$ from healthy and CHF subjects. For both wake and sleep periods we observe a clear separation between healthy and heart failure groups.

\section{Conclusions}

We have analyzed excursion sequences from healthy and heart failure groups during wake and sleep periods. Our results reveal that excursions can be characterized by stretched distributions with different fitting parameters. We observed that for healthy data wake-sleep differences are more signif- icant than for CHF conditions. Further, the application of two procedures to test the alterations of the fitting parameters, also reveals that the distributions from wake and sleep are quite similar for the heart failure group whereas important differences are observed in healthy data. By means of DFA analysis, we confirm the presence of long-term correlations in excursion sequences from healthy data during wake and sleep periods whereas under pathologic conditions correlations are described by a crossover, indicating that over short scales excursions are close to uncorrelated fluctuations. Our results are in concordance with previous studies which report that wake heartbeat fluctuations are characterized by scaling exponents larger than the sleep exponents and to the white noise regime ( $\alpha=0.5)$ [21]. Finally, our analysis reveals that for healthy data during wake and sleep periods, excursions sequences over short scales are less anticorrelated than CHF excursions, indicating stronger neuroatonomic control under heart failure conditions.

\section{Acknowledgments}

We thank F. Angulo, R. Hernández for fruitful comments and suggestions. This work was partially supported by US-Mexico Foundation for Science (FUMEC), EDI-IPN, COFAA-IPN and Consejo Nacional de Ciencia y Tecnología (CONACYT), México.
1. A.L. Goldberger, L A.N. Amaral, J.M. Hausdorff, P.C.h. Ivanov, C.-K. Peng, and H. E. Stanley, PNAS 99 (2002) 2466.

2. G. Sugihara, W. Allan, D. Sobel, and K. Allan, Proc. Nat. Ac. Sci. USA 93 (1996) 2608.

3. P.Ch. Ivanov, L.A.N. Amaral, A.L. Goldberger, S. Havlin, M.G. Rosenblum, Z.R. Stuzik, and H.E. Stanley, Nature 399 (1999) 461.

4. N. Iyengar, C.-K. Peng, R. Morin, A.L. Goldberger, and L.A. Lipsitz, Am J Physiol Regul Integr Comp Physiol 271 (1996) R1078.

5. L. Guzmán-Vargas and F. Angulo-Brown, Phys. Rev. E 67 (2003) 052901.

6. L. Guzmán-Vargas, A. Muñoz-Diosdado, and F. AnguloBrown, Physica A: Statistical Mechanics and its Applications 348 (2005) 304.

7. L.A.N. Amaral, A.L. Goldberger, P. Ch. Ivanov, and H.E. Stanley, Phys. Rev. Lett 81 (1998) 2388.

8. L.A.N. Amaral, P.Ch. Ivanov, N. Aoyagi, I. Hidaka, S. Tomono, A.L. Goldberger, H.E. Stanley, and Y. Yamamoto, Phys. Rev. Lett 86 (2001) 6026.

9. P.Ch. Ivanov, L.A.N. Amaral, A.L. Goldberger, and H.E. Stanley, EPL (Europhysics Letters) 43 (1998) 363.
10. P. Bernaola-Galvan, P.Ch. Ivanov, L.A.N. Amaral, and H.E. Stanley, Phys. Rev. Lett 87 (2001) 168105.

11. M. Ding and W. Yang, Phys. Rev. E 52 (1995) 207.

12. L.S. Liebovitch and W. Yang, Phys. Rev. E 56 (1997) 4557.

13. F.Z. Wang, Y. Kazuko, S. Havlin and H.E. Stanley, Phys. Rev. E 73 (2006) 026117.

14. P. Carpena, P. Bernaola-Galván, P.Ch. Ivanov and H.E. Stanley, Nature 418 (2002) 955.

15. G.F. Newell and M. Rosenblatt, The Annals of Mathematical Statistics 33 (1962) 1306.

16. I. Reyes-Ramírez and L. Guzmán-Vargas, Eur. Phys. Lett. In press (2010)

17. K. Fukuda, H.E. Stanley, and L.A. N. Amaral, Phys. Rev. E 69 (2004) 021108.

18. C.-K. Peng, S. Havlin, H.E. Stanley, and A.L. Goldberger, Phys. Rev. Lett. 70 (1993) 1343.

19. C.-K. Peng, J. Mietus, J.M. Hausdorff, S. Havlin, H.E. Stanley, and A.L. Goldberger, Chaos 5 (1995) 82.

20. A.L. Goldberger et al., Circulation 101 (2000) e215.

21. P. Ch. Ivanov, L.A.N. Amaral, A.L. Goldberger, and H.E. Stanley, EPL (Europhysics Letters) 48 (1999) 594. 